Atmos. Chem. Phys., 3, 1285-1291, 2003

www.atmos-chem-phys.org/acp/3/1285/

\title{
Averaging kernels for DOAS total-column satellite retrievals
}

\author{
H. J. Eskes and K. F. Boersma \\ Royal Netherlands Meteorological Institute, De Bilt, The Netherlands \\ Received: 6 January 2003 - Published in Atmos. Chem. Phys. Discuss.: 18 February 2003 \\ Revised: 30 May 2003 - Accepted: 7 August 2003 - Published: 3 September 2003
}

\begin{abstract}
The Differential Optical Absorption Spectroscopy (DOAS) method is used extensively to retrieve total column amounts of trace gases based on UV-visible measurements of satellite spectrometers, such as ERS-2 GOME. In practice the sensitivity of the instrument to the tracer density is strongly height dependent, especially in the troposphere. The resulting tracer profile dependence may introduce large systematic errors in the retrieved columns that are difficult to quantify without proper additional information, as provided by the averaging kernel (AK). In this paper we discuss the DOAS retrieval method in the context of the general retrieval theory as developed by Rodgers. An expression is derived for the DOAS AK for optically thin absorbers. It is shown that the comparison with 3D chemistry-transport models and independent profile measurements, based on averaging kernels, is no longer influenced by errors resulting from a priori profile assumptions. The availability of averaging kernel information as part of the total column retrieval product is important for the interpretation of the observations, and for applications like chemical data assimilation and detailed satellite validation studies.
\end{abstract}

\section{Introduction}

The Global Ozone Monitoring Experiment (GOME) spectrometer on ESA ERS-2 (Burrows et al., 1999) has demonstrated the unique ability to observe trace gases in the troposphere, including the boundary layer. GOME observes several key species of tropospheric chemistry, such as $\mathrm{O}_{3}$ (Von Bargen and Thomas, 1999; Valks et al., 2002), $\mathrm{NO}_{2}$ (Leue et al., 2001; Richter and Burrows, 2002), HCHO (Chance et al., 2000), BrO (Hegels et al., 1998; Wagner and Platt, 1998; Richter et al., 1998; Van Roozendael et al., 1999) and $\mathrm{SO}_{2}$ (Eisinger and Burrows, 1998). Column amounts of

Correspondence to: H. J. Eskes (eskes@knmi.nl) these tracers have been retrieved using the DOAS technique (Platt, 1994). These data sets contain important information on aspects like fossil fuel burning emissions, natural hydrocarbon emission, biomass burning, $\mathrm{NO}_{\mathrm{x}}$ produced by lightning, and volcano emissions.

The derivation of quantitative tropospheric column amounts for these species is complicated and remains a major challenge. The retrieval depends strongly on aspects like clouds, the assumed profile shape, surface albedo, the presence of a stratospheric background and aerosols. All these aspects can give rise to large errors in the retrieved vertical column for individual measurements.

The DOAS total column retrieval is implicitly dependent on an a priori tracer profile. The radiative transfer calculation in DOAS accounts for the sensitivity of the measurement to tracer concentrations at all altitudes. These sensitivities are implicitly weighted with the assumed tracer profile to produce the retrieved column. The averaging kernel (AK) is proportional to this measurement sensitivity profile, and provides the relation between the retrieved quantities and the true tracer profile. The kernel therefore provides important information needed for a quantitative analysis of the satellite data (Rodgers, 2000; Rodgers and Connor, 2003).

The averaging kernel concept is by now well established in remote sensing. Applications are for instance the retrieval of profiles of atmospheric quantities like temperature and tracers like ozone from satellite measurements. Retrieval groups are increasingly including the kernel information in the profile data products disseminated to users. This is in contrast to the retrieval of total column data such as the DOAS products mentioned above, or for instance Total Ozone Mapping Spectrometer (TOMS) total ozone. It is well known for all these retrievals that the sensitivity of the satellite instrument is (strongly) height dependent (e.g. Hudson et al., 1995; Martin et al., 2002b; Lamarque et al., 2002, and the references given above). This profile dependence implies that kernel information is needed to make optimal use of the retrieved 
tracer columns. However, AKs have not been defined in the literature on DOAS, and they are not included in DOAS total column data products.

Recently an improved approach has been introduced (Palmer et al., 2001; Martin et al., 2002a) to address the profile dependence of the retrieval. This consists of a direct coupling of the retrieval with the output of a chemistry-transport model, in which the a priori profile used in the air-mass factor calculation is replaced by a more realistic model-derived time and space dependent profile. In fact, the results described in these papers can also be obtained when averaging kernels are used in the model/satellite inter-comparisons and vertical column reconstructions.

The paper consists of three topics. First, the DOAS approach will be discussed based on the general retrieval formalism developed by Rodgers. In this alternative approach to DOAS averaging kernels arise naturally. Second, for optically thin absorbers an explicit expression of the DOAS AK is derived in terms of the familiar DOAS air-mass factors. Third, in Sect. 4 the application of the AK will be discussed.

\section{Retrieval and averaging kernels}

There is an important difference between in situ and remote sensing (e.g. satellite) observations. The former can normally be interpreted as true point measurements, while retrieved satellite quantities always represent a weighted average over all parts of the atmosphere that contribute to the signal observed by the satellite instrument. The averaging kernel matrix defines the relation between the retrieved quantities and the true atmospheric state. In the derivation below we will closely follow the discussion in the book of Rodgers (Rodgers, 2000), but concentrating on column observations of atmospheric trace gases.

An observation vector $\mathbf{y}$, e.g. a spectrum of reflectivities in the UV-visible, can be related to the true distribution of the trace gas $\mathbf{x}$ by a forward model $\mathbf{F}$,

$\mathbf{y}=\mathbf{F}(\mathbf{x}, \hat{\mathbf{b}})+\Delta \mathbf{F}+\frac{\partial \mathbf{F}}{\partial \mathbf{b}}(\mathbf{b}-\hat{\mathbf{b}})+\epsilon$.

The forward model accounts for the radiative transfer in the atmosphere, and instrument effects. The vector of parameters $\mathbf{b}$ is a subset of all quantities - apart from the trace gas under consideration - that influence the measurement, and $\hat{\mathbf{b}}$ is the best estimate of these forward model parameters. Examples of $\mathbf{b}$ are the satellite observation geometry (solar zenith angle, viewing angle), properties of clouds, surface properties, the presence of other tracers and aerosols, Ring effect, spectral line strengths and instrument aspects. The right-hand side includes three sources of errors: $\epsilon$ is the measurement noise, $\Delta \mathbf{F}$ is the (often systematic) error in the forward model, and $\partial \mathbf{F} / \partial \mathbf{b}(\mathbf{b}-\hat{\mathbf{b}})$ describes the errors resulting from uncertainties in the model parameters $\mathbf{b}$.
For (weakly) non-linear forward models (moderately optically thin absorbers), Eq. (1) can be expanded around an a priori trace gas distribution $\mathbf{x}_{a}$ when $\mathbf{x} \approx \mathbf{x}_{a}$,

$\mathbf{y}=\mathbf{F}\left(\mathbf{x}_{a}, \hat{\mathbf{b}}\right)+\mathbf{K}_{x}\left(\mathbf{x}-\mathbf{x}_{a}\right)+$ error terms.

The term $\mathbf{K}_{x}=\partial \mathbf{F} / \partial \mathbf{x}$ is the weighting function or Jacobian matrix, evaluated at $\mathbf{x}=\mathbf{x}_{a}$

A retrieval method $\mathbf{R}$ computes a vector (or scalar) of estimated trace gas quantities $\hat{\mathbf{x}}$ based on the measured values $\mathbf{y}$, the a priori information and forward model parameters,

$\hat{\mathbf{x}}=\mathbf{R}\left(\mathbf{y}, \mathbf{x}_{a}, \hat{\mathbf{b}}\right)$

Note that the number of elements of the retrieved state $\hat{\mathbf{x}}$ may differ from that of the $\mathbf{x}$. A matrix equation is obtained when this expression is linearised around the a priori state $\mathbf{y}_{a}=\mathbf{F}\left(\mathbf{x}_{a}, \hat{\mathbf{b}}\right)$,

$\hat{\mathbf{x}}=\mathbf{R}\left[\mathbf{F}\left(\mathbf{x}_{a}, \hat{\mathbf{b}}\right), \mathbf{x}_{a}, \hat{\mathbf{b}}\right]+\mathbf{G}_{y}\left[\mathbf{K}_{x}\left(\mathbf{x}-\mathbf{x}_{a}\right)+\right.$ error terms $]$.

Here we have used Eq. (2), and $\mathbf{G}_{y}=\partial \mathbf{R} / \partial \mathbf{y}$. Subtract the a priori $\hat{\mathbf{x}}_{a}$ on both sides $\left(\mathbf{A}=\mathbf{G}_{y} \mathbf{K}_{x}\right)$,

$$
\begin{aligned}
\hat{\mathbf{x}}- & \hat{\mathbf{x}}_{a}=\mathbf{A}\left(\mathbf{x}-\mathbf{x}_{a}\right)+\mathbf{G}_{y}[\Delta \mathbf{F}+\partial \mathbf{F} / \partial \mathbf{b}(\mathbf{b}-\hat{\mathbf{b}})+\epsilon] \\
& +\mathbf{R}\left[\mathbf{F}\left(\mathbf{x}_{a}, \hat{\mathbf{b}}\right), \mathbf{x}_{a}, \hat{\mathbf{b}}\right]-\hat{\mathbf{x}}_{a} .
\end{aligned}
$$

$\hat{\mathbf{x}}_{a}$ is related to $\mathbf{x}_{a}$ by, for instance, a summation over subsets of vertical layers (see remarks below). For total column retrievals, the a priori column $\hat{x}_{a}=\sum_{l} x_{a, l}$, where $x_{a, l}$ is the a priori sub-column in layer $l$.

\section{Remarks}

1. The first term on the right in Eq. (5) describes the relation between the retrieved quantities and the true distribution of the tracer through the matrix A, called the averaging kernel. For column retrievals the AK is a vector. In the framework of data assimilation the AK is part of the observation operator: a recipe to calculate a forecast of the retrieved observations based on the model state.

2. The other terms describe sources of error related to the forward model and knowledge of the model parameters. The second term on the right describes errors related to the forward model. The last term on the right describes how well the retrieval is able to reproduce the a priori. Normally the retrieval method is constructed in such a way that this is the case, and this last term $=0$. Note that this is not automatically guaranteed for the DOAS retrieval (e.g. for $\mathbf{x}_{a}=0$ ).

3. A discussion of the error sources can be based on Eq. (5), but is beyond the scope of this note (Boersma et al., 2003). 
4. Equation (5) is a generalisation of the discussion by Rodgers (2000). In our derivation the trace gas state vector $\mathbf{x}$ and the vector of retrieved quantities $\hat{\mathbf{x}}$ may be of different dimension. In his book, Rodgers discusses profile retrieval problems with the forward model and retrieval method both defined for the same set of vertical levels, and $\hat{\mathbf{x}}_{a}=\mathbf{x}_{a}$. In practice it will often be natural to choose different sets of vertical layers for the forward model and the retrieval method (this general extension is discussed in Rodgers and Connor, 2003). The total column retrieval is an extreme example of this. A forward model often requires a large number of vertical layers to achieve a good accuracy. In the retrieval the number of levels may reflect the degrees of freedom of signal. One example of this are the ozone profiles provided on "Umkehr" layers in the case of the Solar Backscatter UltraViolet (SBUV) satellite instruments.

\section{DOAS total column retrieval}

In the DOAS approach it is assumed that the reflectivity spectrum can be well approximated by the following equation (Platt, 1994; Burrows et al., 1999),

$\ln R(\lambda) \sim \sum_{t} \sigma_{t}(\lambda) \mathcal{S}_{t}+$ polynomial.

The sum over $t$ is over all tracers that influence the measurement in a predefined spectral window, and may also contain a correction for the Ring effect (inelastic Raman scattering). The remaining spectrum is assumed to be smooth and is approximated by a low-order polynomial of the wavelength $\lambda$. Especially this polynomial is characteristic: the information is obtained from the differential structures and the smooth background is not used in the retrieval.

Based on this approximation, DOAS becomes a two step retrieval approach. The first step is a fit of the differential spectral features with the absorption cross section of the tracer $\sigma_{\lambda}$ to be retrieved. This results in a slant column $\mathcal{S}$ : the effective total column of the tracer along the mean path the light has followed through the atmosphere. Next, an air-mass factor $\mathcal{M}$ is computed that relates the slant column to the vertical column amount $\mathcal{V}=\mathcal{S} / \mathcal{M}$, expressed in Dobson units (ozone) or in molecules $\mathrm{cm}^{-2}$. The slant column fit does not depend directly on a priori information. $\mathcal{M}$ accounts for the atmospheric aspects, and is calculated with a radiative transfer model, based on an a priori profile $\mathbf{x}_{a}$.

In the literature several modifications have been introduced to improve the simple DOAS approximation as discussed above. For instance one may introduce corrections for non-linear effects in the ozone retrieval, the temperature (and therefore height) dependence of the cross sections, the dependence of the air-mass factor on wavelength, Ring effect, and/or inaccuracies resulting from the polynomial. A discussion of such modified DOAS approaches is beyond the scope of this paper. One should be aware that such modifications of DOAS may also introduce changes in the derived expression for the averaging kernel.

In this section we specialise on weak absorbers (trace gases with an absorption optical thickness less than 1). This is a good approximation for the trace gases of interest $\left(\mathrm{NO}_{2}\right.$, $\mathrm{HCHO}, \mathrm{BrO}, \mathrm{OClO}, \mathrm{SO}_{2}$ ) retrieved in the $\mathrm{UV}$ and visible spectral range. For instance $\mathrm{NO}_{2}$ has a cross section in the order of $5 \times 10^{-19} \mathrm{~cm}^{2}$ in the $400-450 \mathrm{~nm}$ range, resulting in a typical absorption optical thickness of the order of 0.005 . Also ozone has an absorption optical thickness $<1$ for the wavelengths at which DOAS is applied (larger than about $320 \mathrm{~nm})$.

The DOAS approach is based on the Beer-BouguerLambert law for the transmission of light over a distance $s$ through an absorbing gas of density $\rho$,

$$
I_{\lambda}(s)=I_{\lambda}(0) \exp \left[-\int_{0}^{s} \sigma(\lambda) \rho d s\right]
$$

Where $I$ is the intensity of the light. For a small optical thickness this exponential form can be approximated by

$$
I_{\lambda}(s)=I_{\lambda}(0)\left[1-\int_{0}^{s} \sigma(\lambda) \rho d s\right]
$$

The intensity of the light in the atmosphere in this small absorption optical thickness limit is linear in the amount of tracer, and the effect of tracer densities at different altitudes on the radiation is additive.

For weak absorbers (such as $\mathrm{NO}_{2}$ ) the forward model can be linearised around $\mathbf{x}_{a}=0$.

$\mathbf{y}=\mathbf{F}(0, \hat{\mathbf{b}})+\mathbf{K}_{x} \mathbf{x}+$ error terms,

where $\mathbf{x}$ is an array of tracer partial columns in the layers defined by the forward model. $\mathbf{F}(0, \hat{\mathbf{b}})$ and $\mathbf{K}_{x}$ no longer depend on $\mathbf{x}$. They do, however, strongly depend on the forward model parameters $\hat{\mathbf{b}}$. The term $\mathbf{F}(0, \hat{\mathbf{b}})$ is dominated by scattering terms (Rayleigh, surface) and absorption by other gases like ozone. The term $\mathbf{K}_{x}$ describes the differential spectral structure, roughly proportional to the absorption cross-section of the trace gas. It specifies the heightdependence of the sensitivity of the spectrum to changes in the tracer concentration.

Similarly, the retrieval method $R$ can be linearised around $\mathbf{F}(0, \hat{\mathbf{b}})$. Note that the retrieval still depends on the a priori tracer profile shape because of the profile dependence of the air-mass factor. We can write $\mathbf{x}_{a}=\lim _{\delta \rightarrow 0} \delta \mathbf{x}_{a}^{0}$, where $\mathbf{x}_{a}^{0}$ represents a non-zero a priori profile that determines the airmass factor $\mathcal{M}$ and $\delta$ is a small number,

$\mathcal{V}=\hat{x}=R\left[\mathbf{F}(0, \hat{\mathbf{b}}), \mathbf{x}_{a}^{0}, \hat{\mathbf{b}}\right]+\mathbf{G}_{y} \mathbf{K}_{x} \mathbf{x}+$ error terms.

With the definition of the averaging kernel, and because $R\left[\mathbf{F}(0, \hat{\mathbf{b}}), \mathbf{x}_{a}^{0}, \hat{\mathbf{b}}\right] \approx 0$,

$\mathcal{V}=\mathbf{A} \mathbf{x}+$ error terms. 


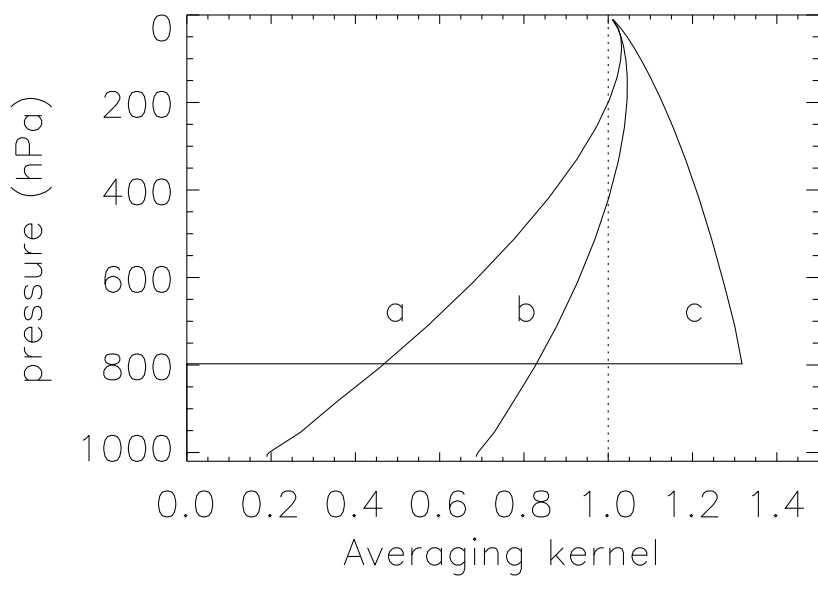

Fig. 1. Example of DOAS averaging kernels at $437 \mathrm{~nm}$ : (a) clear pixel with a surface albedo of 0.02 ; (b) clear pixel with a surface albedo of 0.15 ; (c) pixel with an optically thick cloud and cloud top at $800 \mathrm{hPa}$.

In terms of the two DOAS steps,

$$
\begin{aligned}
\mathcal{V}=R\left(\mathbf{y}, \hat{\mathbf{b}}, \mathbf{x}_{a}^{0}\right)=\mathcal{S} / \mathcal{M}\left(\mathbf{x}_{a}^{0}, \tilde{\mathbf{b}}\right), \\
\quad \mathbf{G}_{y}=\partial R / \partial \mathbf{y}=1 / \mathcal{M}\left(\mathbf{x}_{a}^{0}, \tilde{\mathbf{b}}\right) \partial \mathcal{S} / \partial \mathbf{y} .
\end{aligned}
$$

Here $\tilde{\mathbf{b}}$ is the subset of the parameters $\hat{\mathbf{b}}$ that describe atmospheric aspects. In a similar way the forward model may be approximated,

$\mathbf{y}=\mathbf{F}(\mathbf{x}, \hat{\mathbf{b}}) \approx \mathbf{Y}[S(\mathbf{x}, \tilde{\mathbf{b}}), \hat{\mathbf{b}}], \mathbf{K}_{x}=\partial \mathbf{Y} / \partial S \partial S / \partial \mathbf{x}$.

The operator $S(\mathbf{x}, \tilde{\mathbf{b}})$ in Eq. (13) (to be distinguished from the retrieved $\mathcal{S}$ ) computes a slant column based on the tracer profile, and $\mathbf{Y}$ computes a spectrum based on $S$.

The two-step approximation assumes that the dependence of the spectrum on the tracer distribution can be described by a single quantity, namely the slant column. One important exception to this is the temperature-dependence of the cross section. This introduces an indirect profile dependence. Equation (13) assumes that this dependence is small and can be neglected (or that an efficient a posteriori correction can be applied).

Normally one will assume that the DOAS fit of the retrieval method accurately reproduces the slant column in the forward model, then $\partial \mathcal{S} / \partial \mathbf{y} \partial \mathbf{Y} / \partial S \approx 1$. In this case the averaging kernel can be directly expressed in terms of the air-mass factor. For an ideal DOAS retrieval this equation will hold. In reality there will be many reasons which will cause this relation to hold only approximately. Sources of errors are inaccuracies in the modelled reflectivities, inaccurate instrument slit function, non-orthogonality of the cross section with other trace gas cross sections and the polynomial, Ring effect, assumptions about the mean temperature of the trace gas and others. If for some reason this relation does not hold, one may want to improve the DOAS approach, instead of introducing a correction factor in the averaging kernel.
As mentioned above, for optically thin absorbers the reflectivities and the slant column are linear with respect to the tracer amounts and can be expressed as a sum of the contributions of each of the layers. One may generally write $S=\sum_{l} C_{l} x_{l}$, with yet unspecified coefficients $C_{l}$. The air mass factor is computed with the relation $M=S / V=S / \sum_{l} x_{l}$. When the tracer is confined to layer $l$, this becomes $M=S / x_{l} \approx \partial S / \partial x_{l}$.

$\frac{\partial S}{\partial x_{l}}=C_{l}=M\left(\epsilon \mathbf{e}_{l}, \tilde{\mathbf{b}}\right)$.

Therefore $C_{l}$ is identified as the air-mass factor $M$ for layer $l$ computed with the radiative transfer model. This is obtained in the same way as the total air-mass factor, but for an optically thin amount $\epsilon$ of tracer confined to layer $l$ only (the unit vector $\mathbf{e}_{l}$ is $=1$ for index $l$, and $=0$ elsewhere).

The elements of the averaging kernel vector $A_{l}$ are the ratio of the air-mass factor of layer $l$ and the total air-mass factor computed from the a priori profile,

$A_{l}=\left[\mathbf{G}_{y} \mathbf{K}_{x}\right]_{l}=\frac{M\left(\epsilon \mathbf{e}_{l}, \tilde{\mathbf{b}}\right)}{M\left(\mathbf{x}_{a}^{0}, \tilde{\mathbf{b}}\right)}$.

The air-mass factor of layer $l$ can be identified as the Jacobian of the forward model $\partial S / \partial \mathbf{x}$. This term determines the height dependence of the averaging kernel and is independent of the tracer distribution for optically thin absorbers. For stronger absorbers the forward model will become non-linear and the Jacobian will depend on $\mathbf{x}$. In this case the appropriate starting point is Eq. (5) which describes the retrieval in terms of departures from the a priori $\mathbf{x}_{a}$.

Figure 1 shows examples of the $\mathrm{AK}$ computed for the $\mathrm{NO}_{2}$ retrieval. The actual shape of the kernel changes from one ground pixel to the next, depending in particular on the presence of clouds, aerosols and on the surface albedo of the scene. For this illustration a simplified a priori profile $\mathbf{x}_{a}^{0}$ is used which peaks in the middle/upper stratosphere, where the air-mass factor approaches the simple geometrical expression $A=1 / \cos \left(\theta^{s z a}\right)+1 / \cos \left(\theta^{v}\right)$. The solar zenith angle $\theta^{s z a}$ is 45 degree and the viewing angle $\theta^{v}$ is zero (nadir view) in the examples. The air-mass factor is calculated at $437 \mathrm{~nm}$.

For curve $\mathrm{c}$ we have approximated the cloud top of an optically thick cloud by a Lambertian reflector. This description of clouds is crude, but it is consistent with assumptions that are often used in cloud retrieval algorithms (e.g. the Fresco algorithm, Koelemeijer et al., 2001) that are used in the DOAS retrieval. These cloud algorithms will retrieve an effective, light path derived, cloud top, which can be expected to be somewhat lower than the actual cloud top.

The figure demonstrates the large range of sensitivities between $200 \mathrm{hPa}$ and $1000 \mathrm{hPa}$, and the low sensitivity in the lower troposphere for cloud free pixels with a dark surface. Because of the large variability of the short-lived $\mathrm{NO}_{2}$ compound in the (lower) troposphere, and because of the presence of $\mathrm{NO}_{2}$ in the stratosphere, $\mathrm{NO}_{2}$ profile shapes show a 
similar large variability. Generally there is a large difference between the sensitivities of the retrieval in the stratosphere as compared to the lower troposphere. For instance, curve (a) in the figure shows roughly a factor 3 difference of the air-mass factor between boundary layer and stratospheric $\mathrm{NO}_{2}$. Since the retrieved vertical column is directly proportional to the air-mass factor, this profile variability implies a large profile dependence of the vertical column retrieved. As a result, errors of the order of $100 \%$ can be expected in the retrieved total column for individual pixels for simplified a priori profile assumptions.

\section{Use of averaging kernel information}

There is one important aspect related to averaging kernels as discussed above. As mentioned, the retrieved total column $\mathcal{V}$ depends on the a priori profile shape. In contrast, a comparison of the DOAS retrieved $\mathcal{V}$ with profile information $\mathbf{x}$ from models or other measurements, based on the averaging kernels (Eq. 11), is independent of the a priori-profile for optically thin absorbers. The air-mass factor in the retrieval of the vertical column, Eq. (12) cancels against the air-mass factor in the denominator of Eq. (15). The averaging kernel describes the sensitivity of the depth of the absorption features in the measured spectra (the slant column) to changes in tracer concentrations at a given altitude (given $\hat{\mathbf{b}}$ is known accurately). It contains the information needed to understand the relation between the retrieved vertical column and the DOAS slant column.

This aspect also influences the error estimation (Boersma et al., 2003). Two observation errors are to be distinguished [related to the smoothing error concept (Rodgers, 2000)]: the observation error for the column $\mathcal{V}$ and the total observation error related to Eq. (11):

1. The total error related to the estimate of the column $\mathcal{V}=\mathcal{S} / M$ consists of slant column measurement errors and air-mass factor errors. The latter consists of errors related to uncertainties in the assumed profile $\mathbf{x}_{a}^{0}$ and errors related to the parameters $\tilde{\mathbf{b}}$.

2. When the retrieval is compared with independent data using Eq. (11), the total observation error consists of a contribution due to the error in $\mathcal{V}$ and a "representativeness" error contribution due to errors in the computation of the kernel. Because the total air-mass factor enters as a denominator in both $\mathcal{V}$ and $\mathbf{A}$ there is a cancellation and $M$ does not contribute to the error.

The total observation error (e.g. as in data assimilation) for the comparison based on averaging kernels now has contributions related to slant column errors and contributions related to parameter errors (errors in the kernel $\mathbf{A}$ ). The a priori-profile error does not influence the comparison, and the total observation error for the comparison based on kernels is smaller than the total observation error in the retrieved column $\mathcal{V}$.

There are two ways to use the DOAS retrievals. Firstly, when the user does not have independent information on the tracer profile, the retrieved $\mathcal{V}$ can be used as the best estimate of the tracer column. The user should be aware of the dependence of this total column on the a priori-profile used in the air-mass factor calculation. Secondly, when profile information is available equation 11 should be used which specifies the relation between $\mathcal{V}$ and the true profile $\mathbf{x}_{a}$ of the tracer:

1. Data assimilation. A successful assimilation of satellite measurements requires accurate observation operators. The relation $\hat{\mathbf{x}}-\hat{\mathbf{x}}_{a}=\mathbf{A}\left(\mathbf{x}-\mathbf{x}_{a}\right)$ describes how the model state $\mathbf{x}$ (e.g. a model $\mathrm{NO}_{2}$ profile) should be related to the retrieved quantities $\hat{\mathbf{x}}$ (the retrieved DOAS $\mathrm{NO}_{2}$ column).

2. Comparisons with model simulations. The averaging kernels allow for a direct comparison between model results and the observations. When the averaging kernels are used, this comparison is no longer complicated by systematic biases caused by unrealistic a priori assumptions (as was shown above). Effectively the GOME slant column is then directly compared with a modelled slant column.

3. Interpretation of the retrieved columns. The averaging kernels describe the sensitivity of the satellite observations to the trace gas profile. This is valuable additional information to understand the measurements. An example is an area with large boundary layer concentrations related to local emissions: despite the large vertical column, the satellite retrieval may show small values of $\mathcal{V}$ at such locations. This may be related to a small sensitivity near the surface in combination with unrealistic a priori profile information.

4. Detailed validation of the satellite retrievals. If additional profile information is available from independent (ground-based) measurements, the averaging kernel relates this local profile to collocated satellite column observations (Rodgers and Connor, 2003).

The derivatives of the forward model with respect to the state or the retrieval parameters (the Jacobians) play a central role in all retrievals. Because of this, most modern radiative transfer codes contain efficient subroutines to compute these Jacobians. For operational use these Jacobians can for instance be stored in look-up tables. The extra effort of providing the averaging kernel information will therefore often be small. For DOAS the averaging kernel is the ratio of two air-mass factors, Eq. (15), and can be computed with the existing DOAS software. 


\section{Conclusions}

The averaging kernel provides a direct interpretation of the satellite retrieval to users. In particular, the AK quantifies the contribution of the stratosphere, troposphere and boundary layer to the observation, depending on aspects like clouds, surface albedo, viewing and solar zenith angle. It also provides an interpretation of the value of the air-mass factor. The use of the AK together with the retrieved column removes the (often large) dependence of the inter-comparison with independent data on a priori assumptions about the profile shape.

The AK provides an alternative way of implementing the combined retrieval/modelling approach described by Palmer et al. (2001); Martin et al. (2002a) The AK is proportional to the height-dependent sensitivity of the satellite observation to changes in the tracer concentration. This is the central information needed to combine model output with the observations. There are two reasons why providing averaging kernels may be preferred. First, the averaging kernel is a well established concept in the retrieval of remote sensing observations, as the link between the retrieved quantities and reality. Secondly, kernels can be provided by retrieval and instrument teams without the direct involvement of 3D chemistry-transport models. The retrieval depends on a detailed knowledge of the instrument aspects, (time-dependent) calibration procedure and knowledge of the radiative transfer, and therefore instrument teams in general are in the best position to perform these retrievals.

The inclusion of kernels (vectors) in the data product will increase the data volume. This is a point of concern: future instruments like for instance Ozone Monitoring Instrument (OMI) will generate large amounts of data. However, in our retrieval product the kernel related data fields are about $40 \%$ of the total data volume which is not unacceptable.

With this paper we hope to stimulate retrieval groups to routinely include averaging kernel information in the data products of GOME and new satellite instruments like SCIAMACHY on Envisat and OMI on EOS-Aura. The above discussion is more general than the application to DOAS only. Averaging kernel information (related to the Jacobians) can be provided for alternative column retrieval approaches such as, for instance, the TOMS total ozone algorithm. The additional kernel information is important for remote sensing retrievals in general, and for tropospheric trace gas retrievals in particular.

Acknowledgements. The authors acknowledge stimulating discussions with R. van Oss.

\section{References}

Boersma, K. F., Eskes, H. J., and Brinksma, E.: Error analysis for tropospheric $\mathrm{NO}_{2}$ retrievals from space, preprint, 2003.

Burrows, J. P., Weber, M., Buchwitz, M., Rozanov, V., LadstätterWeibenmayer, A., Richter, A., Debeek, R., Hoogen, R., Bramstedt, K., Eichmann, K.-U., Eisinger, M., and Perner, D.: The Global Ozone Monitoring Exeriment (GOME): Mission concept and first scientific results, J. Atmos. Sci., 56, 151-175, 1999.

Chance, K., Palmer, P., Spurr, R. J. D., Martin, R. V., Kurosu, T. P., and Jacob, D. J.: Satellite observations of formaldehyde over North America from GOME, Geophys. Res. Lett., 27, 34613464, 2000.

Eisinger, M. and Burrows, J. P.: Tropospheric Sulfur Dioxide observed by the ERS-2 GOME Instrument, Geophys. Res. Lett., 25, 4177-4180, 1998.

Hegels, E., Crutzen, P. J., Klüpfel, T., Perner, D., and Burrows, J. P.: Global distribution of atmospheric bromine monoxide from GOME on Earth observing satellite ERS-2, Geophys. Res. Lett., 25, 3127-3130, 1998.

Hudson, R. D., Kim, J.-H., and Thompson, A. M.: On the derivation of tropospheric column ozone from radiances measured by the total ozone mapping spectrometer, J. Geophys. Res., 100, 11 137-11 145, 1995.

Koelemeijer, R. B. A., Stammes, P., Hovenier, J. W., and de Haan, J. F.: A fast method for retrieval of cloud parameters using oxygen A-and measurements from the Global Ozone Monitoring Experiment, J. Geophys. Res. 106, 3475-3490, 2001.

Lamarque, B. V., Khattatov, J. C., and Gille, J.-F.: Constraining tropospheric ozone column through data assimilation, J. Geophys. Res., 107, doi:10.1029/2001JD001249, 2002.

Leue, C., Wenig, M., Wagner, T., Klimm, O., Platt, U., and Jähne, B.: Quantitative analysis of $\mathrm{NO}_{\mathrm{x}}$ emissions from Global Ozone Monitoring Experiment satellite image sequences, J. Geophys. Res., 106, 5493-5505, 2001.

Martin, R. V., Chance, K., Jacob, D. J., Kurosu, T. P., Spurr, R. J. D., Bucsela, E., Gleason, J. F., Palmer, P. I., Bey, I., Fiori, A. M., Li, Q., and Yantosca, R. M., and Koelemeijer, R.B.A.: An improved retrieval of tropospheric nitrogen dioxide from GOME, J. Geophys. Res., 107, D20, 4437, 10.1029/2001JD001027, 2002.

Martin, R. V., Jacob, D. J., Logan, J. A., Bey, I., Yantosca, R. M., Staudt, A. C., Li, Q., Fiore, A. M., Duncan, B. N., Liu, H., Ginoux, P., and Thouret, V.: Interpretation of TOMS Observations of Tropical Tropospheric Ozone with a Global Model and In-situ Observations, J. Geophys. Res., 107(D18), 4351, doi:10.1029/2001JD001480, 2002.

Palmer, P. I., Jacob, D. J., Chance, K., Martin, R. V., Spurr, R. J. D., Kurosu, T. P., Bey, I., Yantosca, R., Fiore, A., and Li, Q.: Air-mass factor formulation for spectroscopic measurements from satellites: application to formaldehyde retrievals fom the Global Ozone Monitoring Experiment, J. Geophys. Res., 106, 14 539-14 550, 2001.

Platt, U.: Differential optical absorption spectroscopy (DOAS), in Air Monitoring by Spectroscopic Techniques, M.W. Sigrist, ed., Chemical Analysis Series, Wiley, New York, 127, 27-84, 1994.

Richter, A. and Burrows, J. P.: Tropospheric $\mathrm{NO}_{2}$ from GOME measurements, Adv. Space Res., 29, 1673-1683, 2002. 
Richter, A., Wittrock, F., Eisinger, M., and Burrows, J. P.: GOME observations of tropospheric BrO in Northern Hemispheric spring and summer 1997, Geophys. Res. Lett., 25, $2683-$ 2686, 1998

Rodgers, C. D.: Inverse methods for atmospheric sounding - theory and practice, Series on Atmospheric, Oceanic and Planetary Physics, World Scientific Publishing, Singapore, 2000.

Rodgers, C. D. and Connor, B. J.: Intercomparison of Remote Sounding Instruments, J. Geophys. Res., 108, doi:10.1029/2002JD002299, 2003.

Valks, P. J. M., Piters, A. J. M., Lambert, J.-C., Zehner, C., and Kelder, H.: A Fast Delivery System for the retrieval of near-real time ozone columns from GOME data, Int. J. Rem. Sens., 24, 423-436, 2002.
Van Roozendael, M., Fayt, C., Lambert, J.-C., Pundt, I., Wagner, T. et al.: Development of a bromine oxide product from GOME, European Symposium on Atmosph. Measurements from Space, ESA WPP-161, 543-547, 1999.

Von Bargen, A. and Thomas, W.: GOME GDP Update Report for GDP 0-to-1 Version 2.0 and GDP 1-to-2 Version 2.7, ER-TNDLR-GO-0043 (Issue 1/A), DLR/DFD, Oberpfaffenhofen, Germany, 1999.

Wagner, T. and Platt, U.: Satellite mapping of enhancd BrO concentrations in the troposphere, Nature, 395, 486-490, 1998. 